\title{
ESTIMATES ON SINGULAR VALUES OF FUNCTIONS OF PERTURBED OPERATORS
}

\section{QINBO LIU}

Abstract. This is a conitunation of [1] and [2]. We prove that if function $f$ belongs to the class $\Lambda_{\omega} \stackrel{\text { def }}{=}\left\{f: \omega_{f}(\delta) \leqslant\right.$ const $\left.\omega(\delta)\right\}$ for an arbitrary modulus of continuity $\omega$, then $s_{j}(f(A)-$ $f(B)) \leqslant c \cdot \omega_{*}\left((1+j)^{-\frac{1}{p}}\|A-B\|_{S_{p}^{l}}\right) \cdot\|f\|_{\Lambda_{\omega}}$ for arbitrary self-adjoint operators $A, B$ and all $1 \leqslant j \leqslant l$, where $\omega_{*}(x) \stackrel{\text { def }}{=} x \int_{x}^{\infty} \frac{\omega(t)}{t^{2}} d t(x>0)$. The result is then generalized for contractions, maximal dissipative operators, normal operators and $n$-tuples of commuting self-adjoint operators.

Mathematics subject classification (2010): 47A10, 47A55.

Keywords and phrases: Singular values; pertubed operators; modulus of continuity.

\section{REFERENCES}

[1] A. B. Aleksandrov, V. V. Peller, Operator Hölder-Zygmund functions, Adv. Math. 224 (2010), 910-966.

[2] A. B. Aleks androv, V. V. Peller, Functions of operator under perturbation of class $S_{p}$, J. Func. Anal. 258 (2010), 3675-3724.

[3] A. B. Aleks androv, V. V. Peller, Functions of perturbed unbounded self-adjoint operators. Operator Bernstein type inequalities, Indiana Univ. Math. J. 59:4 (2010), 1451-1490.

[4] A. B. Aleks Androv, V. V. Peller, Functions of perturbed dissipative operators, Algebra i Analiz 23 (2011), 9-51; translation in St. Petersburg Math. J. 23 (2012), 209-238.

[5] A. B. Aleksandrov, V. V. Peller, D. Potapov and F. Sukochev, Functions of normal operators under perturbations, Adv. Math. 226 (2011), 5216-5251.

[6] A. Zygmund, Trigonometric series, 2nd ed., vols. I, II, Cambridge University Press, New York, 1959.

[7] F. L. NaZarov, V. V. Peller, Functions of $n$-tuples of commuting self-adjoint operators, J. Funct. Anal. 266 (2014), 5398-5428.

[8] M. S. Birman, M. Z. Solomyak, Double Stieltjes operator integrals, III, Problems of Math. Phys., Leningrad. Univ. 6 (1973), 27-53 (Russian).

[9] M. S. Birman, M. Z. SolomyaK, Spectral theory of selfadjoint operators in Hilbert spaces, Mathematics and its Applications (Soviet Series), D. Reidel Publishing Co., Dordrecht, 1987.

[10] V. V. PeLleR, Hankel operators of class $S_{p}$ and their applications (rational approximation, Gaussian processes, the problem of majorizing operators), Mat. Sbornik, 113 (1980), 538-581 (Russian), English Transl. in Math. USSR Sbornik, 41 (1982), 443-479.

[11] V. V. Peller, Hankel operators in theory of perturbations of unitary and self-adjoint operators, Funktsional. Anal. i Prilozhen. 19:2 (1985), 37-51 (Russian), English Transl. in Funct. Anal. Appl. 19 (1985), 111-123.

[12] V. V. Peller, For which $f$ does $A-B \in S_{p}$ imply that $f(A)-f(B) \in S_{p}$ ?, Operator Theory, Birkhauser, 24 (1987), 289-294.

[13] V. V. PELLER, Hankel operators in the perturbation theory of unbounded self-adjoint operators, Analysis and partial differential equations, 529-544, Lecture Notes in Pure and Appl. Math. 122, Dekker, New York, 1990. 